\title{
Muscles Heating Analysis in Sportspeople to Prevent Muscle Injuries using Thermal Images
}

\author{
Brian Meneses-Claudio ${ }^{1}$, Witman Alvarado-Díaz ${ }^{2}$, Fiorella Flores-Medina ${ }^{3}$ \\ Natalia I. Vargas-Cuentas ${ }^{4}$, Avid Roman-Gonzalez ${ }^{5}$ \\ Image Processing Research Laboratory (INTI-Lab) \\ Universidad de Ciencias y Humanidades, Lima, Perú
}

\begin{abstract}
Muscle heating is the process that every athlete follows before any physical activity or sport which are the legs where greater force is exerted and in case a good heating routine is not practiced, the muscles can suffer tears, cramps or fractures due to sudden movements while the muscles are cold. According to the National Institute of Arthritis and Musculoskeletal and Skin Diseases of the United States, the most common injuries occur in the ankles because it is a central point where greater force is exerted by the induced weight of the athletes, in addition if excessive muscle care is important. That is why the evaluation of muscle heating in athletes to prevent muscle damage was raised in this research work, first two thermal images of the before and after heating will be obtained using the FLIR ONE Pro thermal camera following a protocol of distance, position and temperature range, then the images are processed in the MATLAB software to map them in the temperature range and then subtract them to obtain the zones where the temperature variations are found indicating where an adequate heating has been carried out. As a result, the areas where the subtraction of both images was positive were obtained, this new image of the subtraction is superimposed on the real image, showing the real image with the areas where it has proceeded with an optimal heating.
\end{abstract}

Keywords-Thermal image; muscle heating; heat map; muscle injuries, temperature range

\section{INTRODUCTION}

The heating or stretching of the muscles is important when doing any physical activity or sports, as explained in [1], it does not matter that it is for a short time [2] because when practicing any activity, it is required that all the muscles have been respectively activated. In addition, heating is important because it prevents sprains, tears and cramps that could cause fractures.

According to the National Institute of Arthritis and Musculoskeletal and Skin Diseases of the United States [1], the most common lesion is the ankle because it is where the weight of the body is centered and more physical activity is practiced in the body which is not well trained.

The heating consists in performing a variety of exercises that cause the progressive increase of the muscular temperature, it is recommended that the intensity of the exercises increase with the heating means at the beginning you start with low intensity exercises and then with high intensity exercises.
In [2], it explains that they did tests with young people of 13-15 years of age, in both genders, these young people made a muscle heating of 12 minutes, finishing the heating, they made thermal tests and a variation between the $0.43^{\circ} \mathrm{C}$ and $1.13^{\circ} \mathrm{C}$ were obtained, being the optimal temperatures for athletes. The main objective of this research was the verification of muscle heating in the legs, arms, face and neck, where $1.69^{\circ} \mathrm{C}$ was obtained in the arms, face and neck and $0.72^{\circ} \mathrm{C}$ in the legs and feet, standardizing ranges for the practice of sport.

In [3], it indicates that the main damages that the athletes suffer are in the muscular zones, of which they are considered as muscular pathologies that are responsible in the rehabilitation of the skeletons, for that reason, they made a thermographic study to identify the zones of higher temperature sectoring the muscles of greater importance, in addition, classify the muscle damage to know the ranges of the mildest and the most deadly in which it can reach a muscle breakdown.

There are intensity phases of heating that consist of: a) Cardiovascular heating: it is known as full trot because the muscles require to reach a minimum temperature; b) Joint mobility: they are more complete movements following an order, sequence and starting ascending or descending (ankle, knees, hips, shoulders, neck, etc); c) Global Stretches: it is the final stage of a regular heating where the muscles are stretched and previously having a heating, these stretches can be maintained in a specific position of 6 to 12 seconds avoiding pain [4]. In addition, it is important to have a prudent stretch time in heating because it affects the contractile capacity of the muscles [5].

The physical consequences of not doing muscle heating or bad practice of muscle heating are tears, joint injuries, cramps, ligament injuries being progressive and fractures due to sudden movements in sports where high muscle strength is required [6], in addition, knowing that Athletes tend to care for their muscles and that's why you need an adequate heating and a heating routine where all the muscles of the body are stimulated [7].

The main objective of the research work is the evaluation of the heating of muscles in athletes to prevent muscle damage and indicate if another routine of additional heating is required to that already applied. The evaluation is done before and after doing a muscle heating to check if an optimal heating routine has been done. 
The thermal images show the thermal composition of a body or objective, in addition to differentiating in which zones there is a higher temperature index, also, these images can be processed with image processing software because they are compatible. In addition, it is now known that the use of thermal images serves to verify the variation of temperature in internal maladies and to know if in case a body is sufficiently heat to make a physical effort.

The following research work is structured as follows: In section II, the development of thermal image processing before and after the athlete's heating will be presented. In section III, the results will be shown by the 2 thermal images referring to the before and after the heating and the subtraction of the image superimposed in the real image to identify the areas that have been heated. Finally, in section IV, it presents the discussion and conclusions of the research work.

\section{MethodolOGY}

In the methodology, each part of the segmentation of the temperature variation of the images before and after the heating is developed for the prevention of muscular injuries of the leg, which consist of the acquisition of the thermal images before and after the heating, Segmentation of the zones of higher temperature in both images, subtract both images and finally superimpose the subtraction of both images to the real image to obtain where it has been heated and also identify if an optimal heating has been done.

The steps of the system are shown in Fig. 1, where the processes by which the images are submitted are indicated to end with the superposition of the differentiation of the thermal image in the image where the highest temperature is presented.

\section{A. Image Acquisition}

At the stage of image acquisition, a thermal camera called FLIR ONE Pro was used, it is a camera capable of capturing thermal images and compatible with Android and IOS mobile devices. This camera has 2 lenses [8] as shown in Fig. 2, where the upper lens captures images and the lens of the lower camera captures the thermal image. In addition, this device has a software called as its name, where you can process those thermal images received, for example: change the temperature range, change the colors of the heat map and among other things. For the process of capturing thermal images, it used a converter from Connector $\mathrm{C}$ to Micro USB because the thermal camera has a connector $\mathrm{C}$ from factory and, in this case, it used an Android mobile device. In Fig. 2, the thermal camera and the connector $\mathrm{C}$ to Micro USB connector converter are shown.

In the following Table I, the characteristics of the FLIR ONE Pro thermal camera are shown.

The FLIR ONE Pro is a device capable of capturing thermal images, as well as its complement in heat map, it is also capable of viewing at a distance the temperature distribution of complete surfaces where a temperature variation is identified. This camera was born based on an idea to identify electronic problems in industrial equipment because, manually, it took too much time, on the other hand, with thermal imaging, it could identify where the problems were and solve it in a period of less time, currently, is used in the medical field to know the distribution of body temperature as well as for different projects where the temperature variation data is required.

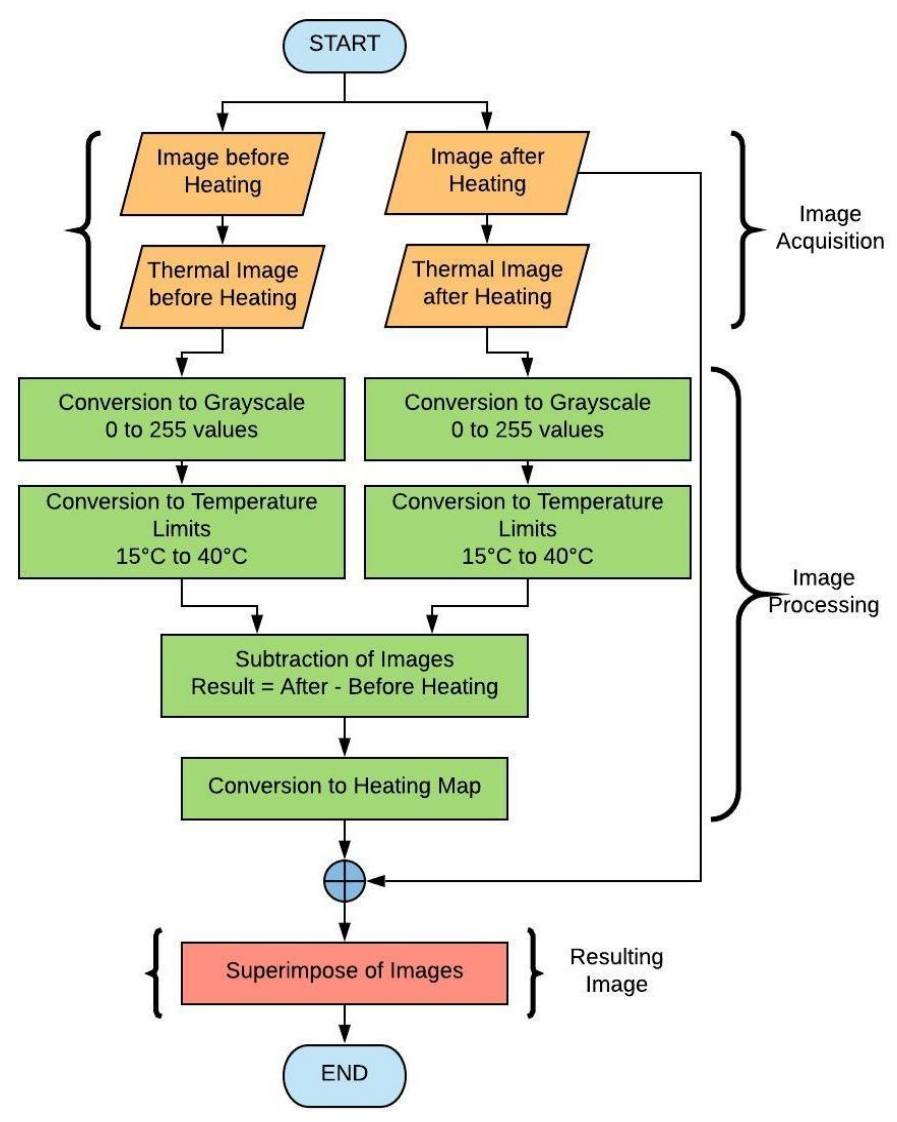

Fig. 1. Flowchart for the Evaluation of Muscle Heating

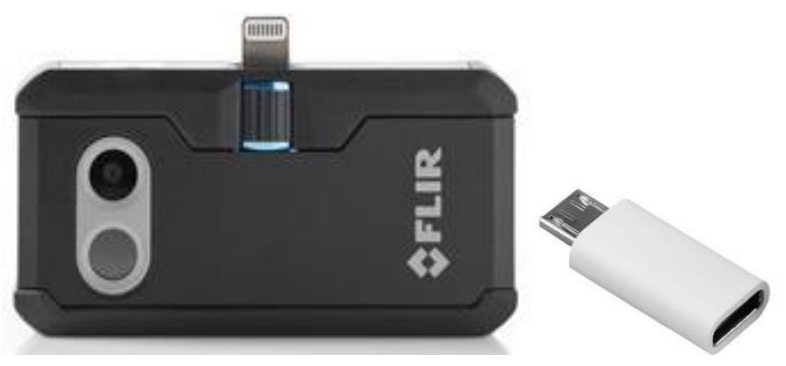

Fig. 2. FLIR ONE Pro Thermal Camera and Type $\mathrm{C}$ to Micro USB Connector Adapter.

TABLE I. CHARACTERISTICS OF THE FLIR ONE PRO

\begin{tabular}{|c|c|}
\hline \multicolumn{2}{|l|}{ FLIR ONE Pro } \\
\hline Temperature Range & $-20^{\circ} \mathrm{C}-400^{\circ} \mathrm{C}$ \\
\hline Compatibility & IOS y Android Devices \\
\hline Maximum Distance & 8 meters \\
\hline Weight & $36.5 \mathrm{~g}$. \\
\hline Dimensions & $68 \times 34 \times 14$ millimeters \\
\hline Thermal Resolution & $160 \times 120$ \\
\hline Work Time & 1 hour \\
\hline
\end{tabular}




\section{B. Image Processing}

The images follow a processing as shown in Fig. 3, where it can observe the thermal images before and after heating, these images are processed according to the flow diagram described above; Fig. 3(d) represents the heat map conversion section, in which the warmest areas of the leg can be observed.

The MATLAB software reads the images received by the FLIR ONE Pro, being the images before and after the heating, each pixel read represented by each dimension.

Then, it converts the thermal images to gray scale so the software uses the following multiplications to give values to each pixel, as shown below:

$0.2989 * R+0.5870 * G+0.114 * B$

This process is done for the thermal images and thus obtain it between the ranges from 0 to 255 in two dimensions. This process is important because in this way it can be converted to a temperature scale.

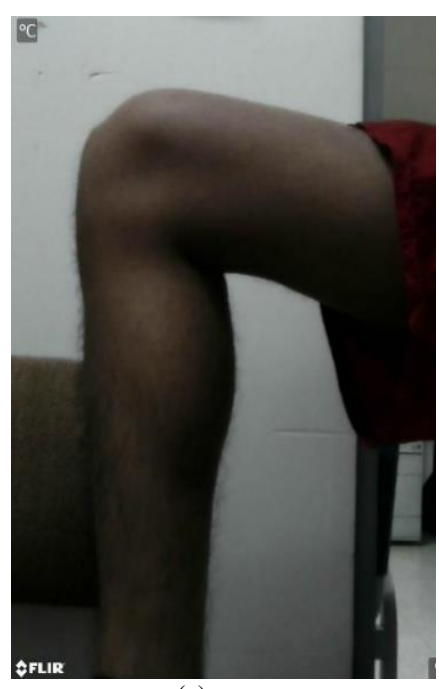

(a)

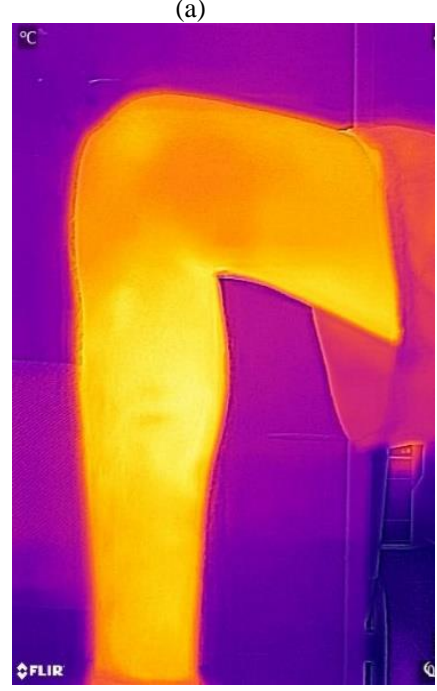

(c)

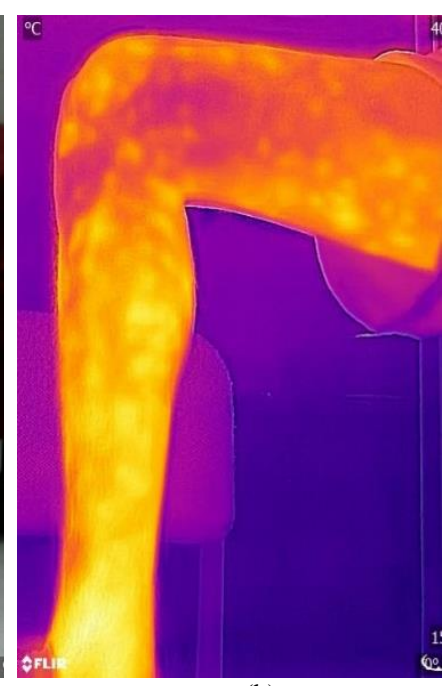

(b)

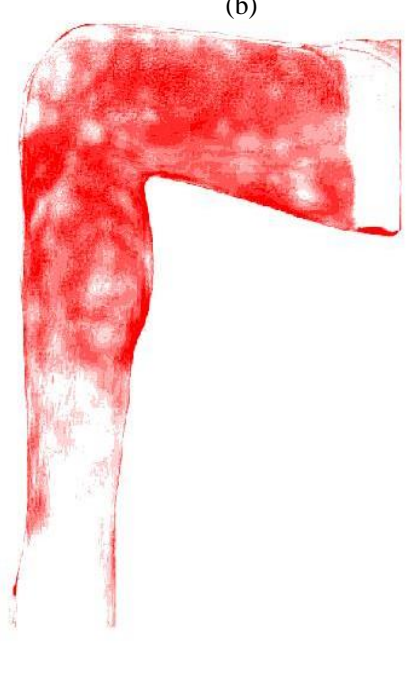

(d)
Fig. 3. (a) Real Image. (b) Thermal Image before Heating. (c) Thermal Image after Heating. (d) Difference of Thermal Images in Heat Map.
Then, convert the images in gray scale to temperature scale with the following formula:

output1 $=(($ Pixel_Image - FromLow $) *($ ToHigh- ToLow $) /$

$($ FromHigh - FromLow $))+$ ToLow

Where:

- FromLow and FromHigh $=0$ y 255 (Gray Scale)

- ToLow and ToHigh $=$ Temperature Range to which will convert the image.

As it wants to convert from gray scale to temperature map, it uses the maximum and minimum ranges in the formula, then get the following formula:

output1 $=(($ Pixel_Image -0$) *(40-15) /(255-0))+15$

Next, it is to subtract the thermal images in temperature scale to obtain the zones where a temperature difference has taken place [9] indicating that it has proceeded with a correct heating. Therefore, a simple subtraction of both thermal images is performed in which the thermal image after heating must predominate.

Subtraction=Thermal_After-Thermal_Before

This result will be converted to heat map, obtaining as a result Fig. 3(d) where the hottest zones are shown and where there has been a more noticeable differentiation of temperature change, in addition, it is identified that some areas have more intense red indicating that it is where the subtraction of temperature is much greater.

Finally, this image will be superimposed on the real image to identify the areas where it has proceeded with a correct heating.

\section{RESULTS}

The thermal images were captured based on a protocol because all the images are required to be homogeneous in that sense, they are captured with the same physical characteristics for further processing. In Table II, the characteristics to which the thermal images were captured are represented.

TABLE II. CHARACTERISTICS OF THERMAL IMAGES

\begin{tabular}{|l|l|}
\hline \multicolumn{2}{|l|}{ Thermal Images } \\
\hline Distance & $40 \mathrm{~cm}$ \\
\hline Temperature Range & $15^{\circ} \mathrm{C}-40{ }^{\circ} \mathrm{C}$ \\
\hline Connected Device & Moto E5 \\
\hline Place where the Image was taken & $\begin{array}{l}- \text { - } \text {-Tibore Tibialis. } \\
\text {-Soleus. }\end{array}$ \\
\hline Rest Time & 0 \\
\hline
\end{tabular}

In [10], the study was conducted in a group of 12 people, divided into 2 randomly selected groups. it was to include heating exercises at the beginning of the training and at the end with the aim of pre-activating (improving the work produced to store elastic energy and using it in later actions) the extensor muscles of the foot and see if they produce difference or improvements in the actions in the game, mainly in the jump. 
The new heating was performed for 3 weeks. Athletes perform heating before training, but rarely, heating helps to pre-activate the muscles. The results obtained were an improvement in the speed of physical actions.

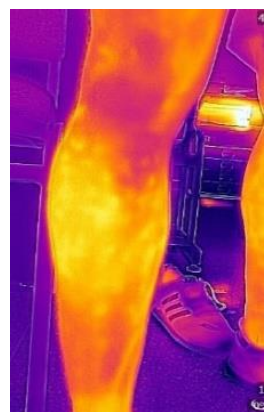

(a)

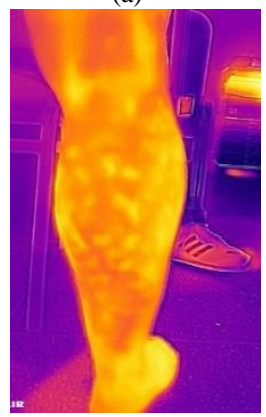

(a)

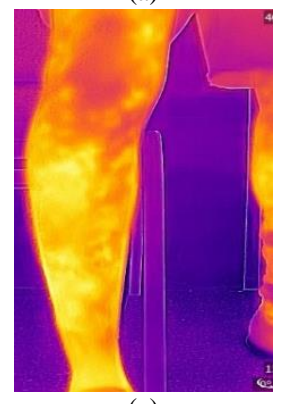

(a)

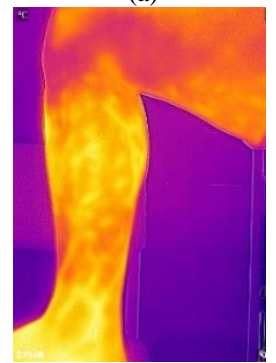

(a)

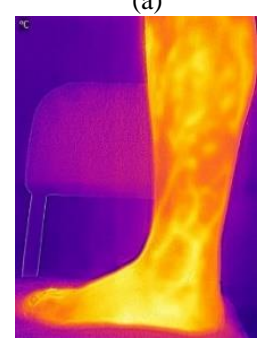

(a)

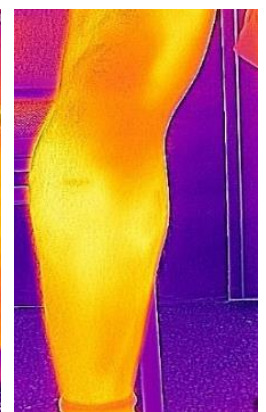

(b)

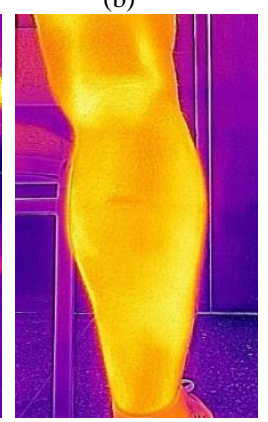

(b)

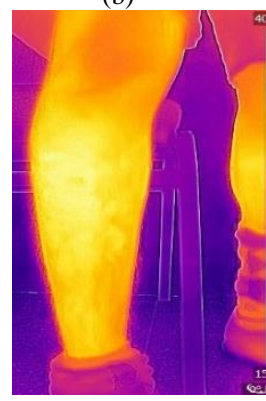

(b)

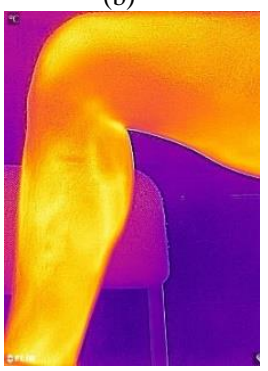

(b)

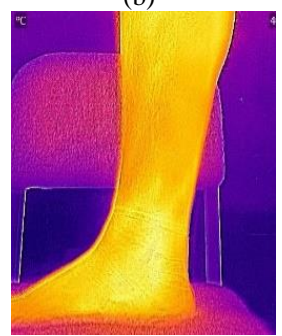

(b)

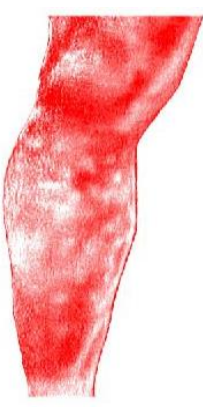

(c)

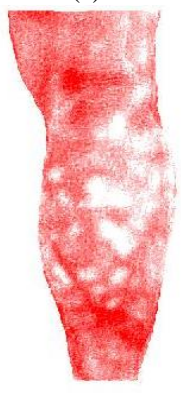

(c)

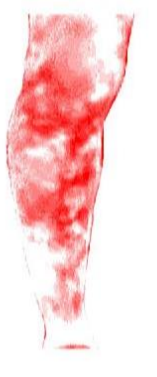

(c)

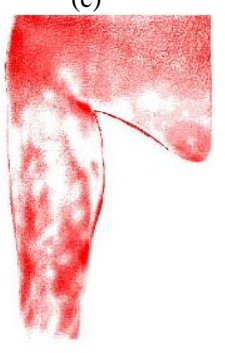

(c)

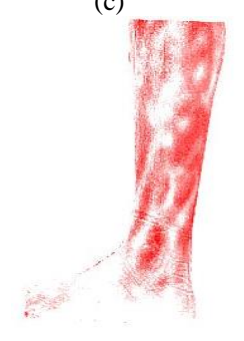

(c)
Fig. 4. (a) Thermal Image before Heating. (b) Thermal Image after Heating. (c) Subtraction of Thermal Images in Heat Map.
In [11], the research tells us about the need for heating and proposes a heating by measuring the type of activity, intensity, recovery time and duration. The author considers the routine of heating very essential to obtain an optimal performance in training or competitions where exercises of power, agility, aerobics and strength should be considered. The heating consists of routine prior to the realization of physical exercise that wants to prepare the athlete to perform to the maximum during training and / or competition. An increase in body temperature causes the glycolytic metabolism and phosphagens in the muscle to be activated more rapidly for a competition; an improvement of the nerve impulse transmission speed is also achieved. There are many studies that indicate that stretching causes very positive effects on performance, but there are 2 that say otherwise these studies indicate that stretching prior to a competition or training harms performance. Except for a stretch of less than 60 seconds (in this case, it does not harm or favor).

Fig. 4 shows the results obtained with a database of 10 soccer athletes, where the thermal images of the before and after heating up will be shown and then the subtraction of the images on the heat map.

\section{DISCUSSION}

The research work confirms the use of thermal images to detect the areas of the body that an athlete must heating, with this information the athlete can avoid injuries due to lack of heating.

The device to which the FLIR ONE Pro will connect is not important because it will only work as thermal image storage because the thermal camera does not contain internal storage.

It tried to capture the thermal images at the precise moment where they had just finished heating up because in several research studies indicate that the human body cools very quickly when no action is taken.

While the reception of thermal images, it needs a protocol where the reader must follow. The protocol must focus on the thermal images and the position where was taken the images.

Some of the sportspeople didn't want to make any analysis because the zone where the images was taken are the most sensible parts of them so it informed about the study and the benefits which brings it.

\section{Conclusions}

It is concluded that the areas with more temperature can be detected through thermal images in an efficient and fast way because only the images need to be captured and then the software will segment it automatically obtaining the zone where the important activated muscles are located for physical activity.

It is concluded that a protocol was established because it was adjusted to the size of the user's leg in addition to having the same characteristics for each image and also to calibrate the FLIR ONE Pro in the required temperature range.

As future work, it wants to create a preventive measure of muscle damage suffered by athletes and also elderly people 
because it knows that their bones and ligaments are worn out over time and could detect muscle damage and follow a healing process avoiding more serious injuries.

As future work, it wants to have a clinic historical of the sportspeople to enforce this study and could improve the performance of them. Also, applying this study when they are training at the gym because there is where they heat all the body and it could sectorized the zones where they don't train.

\section{REFERENCES}

[1] G. Contreras Landgrave, E. J. Camacho Ruiz, S. C. Manzur Quiroga, O. D. Patiño Casas, and L. Ruano Casado, La obesidad en el Estado de México : Interfaces y ocurrencias, vol. 5, no. 1. Universidad Nacional Autónoma de México, Facultad de Estudios Superiores Iztacala, Unidad de Investigación Interdisciplinaria en Ciencias de la Salud y la Educación, 2014.

[2] B. Flores Chico, E. Buendía Lozada, and J. Reynoso Morales, "Dinámica térmica de la piel de adolescentes después del trote," Rev. Ciencias del Ejerc., vol. 10, pp. 16-35, 2016.

[3] M. Schmitt and Y. Guillot, "Thermography and Muscular Injuries in Sports Medicine," in Recent Advances in Medical Thermology, Boston, MA: Springer New York, 1984, pp. 439-445.
[4] B. Meneses-Claudio, W. Alvarado-Diaz, and A. Roman-Gonzalez, "Detection of Suspicions of Varicose Veins in the Legs using Thermal Imaging," 2019.

[5] A. D. Galera, "Orientaciones Didácticas sobre el Calentamiento previo a la Actividad Física," Barcelona, 2018.

[6] C. A. P. Martínez, J. E. F. Soto, and O. A. M. Barrera, "Procedimientos para prevenir las lesiones físicas en la disciplina de Karate-do," Rev. Boletín Redipe, vol. 6, no. 3, pp. 212-219, Mar. 2017.

[7] J. C. Ruíz Quiñonez, "La flexibilidad corporal y su importancia en el desarrollo de actividades físicas / deportivas," Mar. 2019.

[8] S. Agarwal, H. S. Sikchi, S. Rooj, S. Bhattacharya, and A. Routray, "Illumination-invariant Face recognition by fusing thermal and visual images via gradient transfer," Nueva York, Feb. 2019.

[9] J. Azzeh, Z. Alqadi, H. Alhatamleh, and M. Abuzalata, "Creating a Color Map to be used to Convert a Gray Image to Color Image," Int. J. Comput. Appl., vol. 153, no. No 2, pp. 31-34, 2016.

[10] J. M.; Saenz and B. Y. Ureña, "Effect of a Learning Work of the Eccentric-Concentric Cycle on the Jump Capacity in Volleyball.".

[11] A. Pérez-López and D. Valadés, "Bases fisiológicas del calentamiento en voleibol: propuesta práctica (Physiological Basis of Volleyball Warm-Up: Practical Proposal)," Cultura_Ciencia_Deporte, vol. 8, no. 22, pp. 31-40, Mar. 2013. 